In press, Surface Science, (2006)

\title{
Surface-Energy-Anisotropy-Induced Orientation Effects on Rayleigh Instabilities in Sapphire
}

\author{
Melissa K. Santala and Andreas M. Glaeser \\ Ceramic Science Program \\ Lawrence Berkeley National Laboratory \\ $\&$ \\ Department of Materials Science and Engineering \\ University of California \\ Berkeley, CA 94720-1760
}

\begin{abstract}
Arrays of controlled-geometry, semi-infinite pore channels of systematically varied crystallographic orientation were introduced into undoped $\mathbf{m}$-plane $(10 \overline{10})$ sapphire substrates using microfabrication techniques and ion-beam etching and subsequently internalized by solid-state diffusion bonding. A series of anneals at $1700^{\circ} \mathrm{C}$ caused the breakup of these channels into discrete pores via Rayleigh instabilities. In all cases, channels broke up with a characteristic wavelength larger than that expected for a material with isotropic surface energy, reflecting stabilization effects due to surface-energy anisotropy. The breakup wavelength and the time required for complete breakup varied significantly with channel orientation. For most orientations, the instability wavelength for channels of radius $\mathrm{R}$ was in the range of $13.2 \mathrm{R}-25 \mathrm{R}$, and complete breakup occurred within $2-10 \mathrm{~h}$. To first order, the anneal times for complete breakup scale with the square of the breakup wavelength. Channels oriented along a $<11 \overline{2} 0>$ direction had a wavelength of $\approx 139 \mathrm{R}$, and required $468 \mathrm{~h}$ for complete breakup. Cross-sectional analysis of channels oriented along a $<11 \overline{2} 0>$ direction showed the channel to be completely bounded by stable $\mathbf{c}(0001), \mathbf{r}\{\overline{1} 012\}$, and $\mathbf{s}\{10 \overline{1} 1\}$ facets.
\end{abstract}

Keywords: 1) aluminum oxide, 2) lithography, 3) single crystal surfaces, 4) surface energy (anisotropy), 5) Rayleigh instabilities, 6) surface waves - capillary waves, 7) surface structure, morphology, roughness and topography, 8) faceting. 


\section{INTRODUCTION}

Capillary phenomena play an important role in the processing and use of a wide range of materials. Dendritic solidification, sintering of powder compacts, coarsening of catalytic supports, instability of pore networks, diffusive healing of cracks, and the morphologic instability of multilayers, thin films, fibers in composites, and aligned rods in directionally solidified eutectics are examples of processes and situations in which capillarity drives microstructural changes that can either enhance or degrade material performance.

The instability of high-aspect-ratio phases such as fluid jets, wires and rods is among the best known of these phenomena, with the initial stability criterion developed by Plateau in 1856 [1], and the first kinetic analysis by Rayleigh in 1879 [2]. Initially continuous cylindrical fluid jets are prone to the development and growth of periodic axial oscillations in their radius that lead to their breakup into a string of isolated droplets with a characteristic size and spacing. Nichols and Mullins [3,4] extended the modeling of Rayleigh instabilities to solids in the 1960s. Solid rods and cylindrical cavities within solids were predicted to evolve into strings of particles or voids with a size and spacing sensitive to the mass transport mechanism dominating the morphologic evolution. The ability to identify the dominant transport mechanism from the spatial characteristics and to estimate the relevant diffusivity from the temporal characteristics allowed for tracerless measurements of transport coefficients in a wide range of systems.

The analyses of Nichols and Mullins assume an orientation-independent, isotropic specific surface energy. In crystalline solids the specific surface energy often varies with orientation. As a result, equilibrium shapes, rather than being spheres, can be completely or partially facetted, and some unstable high-energy surface orientations can be absent from the equilibrium or Wulff shape $[5,6]$. The specific energies of stable surfaces generally do not vary significantly in a given material, with variations typically on the order of $\pm 10-20 \%$ of the mean surface energy. However, it is not the variation in surface energy per se that matters, but the second derivative of the surface energy with orientation that is critical in dictating the perturbation energetics and kinetically dominant wavelengths [7]. Local surface-energy minima that can stabilize specific crystal facets can also have an enormous stabilizing effect on nonequilibrium morphologies. Thus, in contrast to the behavior of isotropic materials, in which the dominant perturbation 
wavelength is a well-defined multiple of the rod radius, in materials with varying surface energies the details of the surface energy anisotropy (SEA) can vary with the crystallographic orientation of the surfaces and lead to a broad spectrum of evolution behavior within a given material. This presents both processing opportunities and challenges. SEA-induced destabilization may provide opportunities for disruption of continuous phases into finer-scale discrete particles. At the other extreme, every tenfold decrease in the size of a cylindrical fiber or rod can decrease the time required for breakup by a factor of as much as $10^{4}$ (when instability is surface-diffusion or interfacial-diffusion controlled) $[3,4,8]$. Thus, using SEA to stabilize desired nonequilibrium geometries of fine scale structures may become an appealing and perhaps even necessary option.

An extensive program of research spanning two decades has focused on quantifying the SEA of both undoped and doped sapphire (single crystal $\mathrm{Al}_{2} \mathrm{O}_{3}$ ) through determinations of the Wulff shape [912], and on quantifying the effects of SEA on the morphological evolution of internal voids [9, 13-25]. The experimental approach has exploited lithographic processing to produce cavities of controlled geometry in the surfaces of sapphire substrates of known orientation and chemistry. These defects are subsequently internalized by solid-state diffusion bonding [13]. This approach affords a high level of control over the defect geometry, the crystallography of the bounding surfaces, and the chemistry of the enclosing sapphire, the ability to reproduce a structure, and the potential to vary the geometry, crystallography and chemistry independently and systematically. The present paper describes the instability of high-aspect-ratio pore channels etched into an unstable high-energy plane of undoped sapphire, the $\mathrm{m}(10 \overline{10})$ plane, and compares the evolution behavior at $1700^{\circ} \mathrm{C}$ to that obtained in prior work for channels etched into a stable lowenergy plane, the $\mathrm{c}(0001)$ plane, that is part of the Wulff shape of sapphire [23]. The results illustrate the broad spectrum of behaviors that can arise, and identifies opportunities for effective SEA-induced stabilization. 


\section{BACKGROUND}

\section{Modeling Rayleigh Instabilities in Isotropic Systems}

Historically, treatments of the morphologic instability of continuous phases focused on infinite cylinders of radius $\mathrm{R}$ with isotropic specific surface energy $\gamma_{s}$, in which perturbations of infinitesimal amplitude $\alpha_{0}$ and wavelength $\lambda$ are assumed to pre-exist $[1,2]$. Two characteristic quantities emerge from such analyses. The first is a thermodynamic lower limit on the wavelength of growing perturbations, $\lambda_{\min }$, which is independent of the mass-transport mechanism. Perturbations with $\lambda<\lambda_{\min }=2 \pi \mathrm{R}$ are unstable and decay. Growth of an infinitesimal-amplitude perturbation with $\lambda>\lambda_{\min }$ reduces the interfacial area and thus energy per unit length or volume of the rod. Continued amplitude growth ultimately causes breakup of the continuous phase into strings of discrete particles or pores. The second characteristic quantity, $\lambda_{\max }$, defines the expected spacing of these particles or pores by assessing the relationship between $\lambda$ and amplitude growth rate. Perturbations of wavelength $\lambda_{\max }$ increase amplitude most rapidly, and therefore are expected to control the ultimate spacing. For a given transport mechanism, $\lambda_{\max }$ is a fixed multiple of $\lambda_{\min }$, and thus of R [3]. When surface diffusion dominates transport, $\lambda_{\text {max }}=\sqrt{2} \cdot \lambda_{\text {min }}$ $(\approx 8.89 \mathrm{R})$; for lattice-diffusion-controlled breakup of a pore channel, $\lambda_{\max } \approx 1.459 \lambda_{\min }(\approx 12.96 \mathrm{R})$. Thus, in principle, precise measurements of the (spherical) pore size and spacing, or measurements of the pore spacing and knowledge of $\mathrm{R}$ can be used to infer the transport mechanism dominating perturbation growth. These linear stability analyses also provide a means of estimating the relevant diffusivity from observed breakup kinetics.

Models for isotropic systems that consider the presence of finite-amplitude perturbations have been presented, and as the initial amplitude $\left(\alpha_{0}\right)$ increases, $\lambda_{\min }$ decreases [26], the kinetic maximum shifts to higher values of $\lambda$, specifically, $\lambda_{\max }=\left[\sqrt{2}+0.98 \cdot\left(\alpha_{\mathrm{o}} / \mathrm{R}\right)\right] \cdot \lambda_{\min }$, and the time for pinchoff is also affected [27]. For $\alpha_{\mathrm{o}}=0.1 \cdot \mathrm{R}$, the rates indicated by the nonlinear analysis exceed those predicted by the linear analysis, with a $2 \times$ enhancement when $\lambda / \lambda_{\min } \approx \sqrt{2}$, and a $5 \times$ enhancement when $\lambda / \lambda_{\min } \approx 10$. The effect of variations in the perturbation amplitude and interactions between perturbations of differing wavelength on evolution behavior have also been considered [28] in an effort to understand the scatter in the particle spacing, $L$, often seen in experimental studies, and shifts to dominant wavelengths that exceed 
the predicted $\lambda_{\text {max }}$. Pre-existing perturbations with a normalized amplitude $\left(\alpha_{0} / \mathrm{R}\right)$ in the range of 0.01 to 0.05 were found to introduce considerable scatter in $L$, and the average particle/pore spacing, $\bar{L}$, could approach $3 \times$ the value of $\lambda_{\text {max }}$ in isotropic systems.

\section{Modeling Rayleigh Instabilities in Anisotropic Systems}

Even more significant corrections to the behavior predicted by linear models of isotropic solids arise when the effects of SEA are incorporated. Reorientation of the surface owing to perturbation growth can then alter the local surface orientation and energy, and thereby the energetics for wave growth. As a result, the values of $\lambda_{\min }, \lambda_{\max }$, and $\bar{L}$ can be shifted relative to their isotropic counterparts.

Thermodynamic and kinetic analyses of the morphological evolution of pore channels in idealized anisotropic materials by Cahn [7] and by Stölken and Glaeser [29], respectively, have predicted the influence of SEA on $\lambda_{\min }$ and $\lambda_{\text {max }}$, respectively. For the special case of transverse isotropy, and surfacediffusion-controlled evolution, $\lambda_{\max }=\sqrt{2} \cdot \lambda_{\min }$ as in the isotropic case, however, $\lambda_{\min }$ is a function of the surface energy-surface orientation relationship. Specifically,

$$
\lambda_{\text {min }}^{\text {aniso }}=2 \pi \mathrm{R}\left(1+\frac{1}{\gamma_{s}}\left(\frac{\partial^{2} \gamma_{s}}{\partial \phi^{2}}\right)\right)^{\frac{1}{2}}
$$

where $\gamma_{s}$ is the surface energy at the mean channel orientation, $\phi=(\partial \mathrm{R} / \partial z)$ where $z$ is the axial coordinate, and thus $\left(\partial^{2} \gamma_{s} / \partial \phi^{2}\right)$ is related to the curvature of the Wulff plot about the mean orientation. The sign and magnitude of $\left(\partial^{2} \gamma_{s} / \partial \phi^{2}\right)$ are thus predicted to determine the direction and magnitude of the shift in $\bar{L}$ relative to the value of $8.89 \mathrm{R}$ expected in an isotropic system. For an initial orientation that corresponds to a sharp minimum in the $\gamma_{s}-\phi$ plot, $\left(\partial^{2} \gamma_{s} / \partial \phi^{2}\right)$ will be large and positive. As a result, a substantial stabilization would be expected, and $\lambda_{\text {min }}^{\text {aniso }} \gg 2 \pi \mathrm{R}$ is possible. Conversely, when $\left(\partial^{2} \gamma_{s} / \partial \phi^{2}\right)<0, \lambda_{\text {min }}$ will be $<2 \pi \mathrm{R}$. Recently, Gurski and McFadden [30] have extended these considerations to more complex variations of surface energy with orientation.

Glaeser [31] extended the analysis of Stölken and Glaeser [29] to quantify the effect of SEA on the kinetics of evolution. If in the idealized anisotropic system $\lambda_{\max }$ is a factor $k$ times $\lambda_{\text {max }}$ in an isotropic system, i.e., $\lambda_{\max }^{\text {aniso }}=k \lambda_{\max }$, then for surface-diffusion-controlled evolution the evolution time is $k^{2}$ times that 
in an isotropic system. Since values of $k>10$ have been observed [16, 18, 19, 23], anisotropy can cause significant changes in the evolution time. When such experimental observations are interpreted in the context of models for isotropic solids, SEA-induced variations in the evolution times can lead to estimates of the surface diffusivity that can vary considerably, and are in error. From a practical perspective, such stabilization effects could extend the lifetime of fine-scale structures, or allow increases in the use or processing temperature for such structures.

\section{Surface Energy Anisotropy in Sapphire}

In recent years, several studies addressing SEA in sapphire have been performed. These range from studies of surface stability and instability [32-35] to studies in which the equilibrium shape of both undoped and doped sapphire have been determined as a function of temperature $[9-12,22,36]$.

Heffelfinger et al. [33-35] assessed the morphologic stability of vicinal $\mathbf{c}(0001)$ and $\mathbf{m}(10 \overline{10})$ surfaces in undoped sapphire annealed in air at $1400^{\circ} \mathrm{C}$. Vicinal (0001) surfaces formed a terrace-ledge structure, with large $\mathbf{c}(0001)$ terraces, indicating the (0001) surface is stable. In contrast, under similar annealing conditions, the $\mathrm{m}$ plane decomposed into a higher surface area but lower surface energy hill-andvalley structure consisting of $\mathbf{r}\{\overline{1} 012\}$ and $\mathbf{s}\{10 \overline{1} 1\}$ facets. An independent study has shown similar behavior in $\mathrm{m}$-plane sapphire substrates at $1600^{\circ} \mathrm{C}$. Choi et al. [37] used crack healing to generate fine isolated pores in undoped sapphire, and determined their shapes after extended annealing in air at $1600^{\circ} \mathrm{C}$. Kitayama and Glaeser produced arrays of fine pores in undoped sapphire by sequential application of photolithography, ion-beam etching and diffusion bonding, and examined the shape equilibration of these internal pores in response to annealing in flowing gettered argon at $1600^{\circ} \mathrm{C}, 1800^{\circ} \mathrm{C}[12,36]$, and in vacuum at $1900^{\circ} \mathrm{C}[11,36,37]$. Both studies identified the same five facet planes as part of the equilibrium shape at $1600^{\circ} \mathrm{C}: \mathbf{c}(0001), \mathbf{r}\{\overline{1} 012\}, \mathbf{p}\{11 \overline{2} 3\}, \mathbf{s}\{10 \overline{1} 1\}$ and $\mathbf{a}\{11 \overline{2} 0\}$. Since the $\mathbf{m}$ plane is unstable over a wide range of annealing conditions, fundamental differences in the evolution of high-aspect-ratio pore channels etched into $\mathrm{c}$ and $\mathrm{m}$ planes can be anticipated. 


\section{Experimental Studies of Rayleigh Instabilities in Sapphire}

Aluminum oxide is a model ceramic material with a wide range of technological applications. The availability of optically transparent single crystals made the material a convenient system for studies of Rayleigh instabilities. When cracks are introduced by machining, indentation, or partial cleavage of a sample, the cracks undergo a series of morphological changes during high-temperature annealing [38-41]. The cracks evolve into pore channels, which subsequently undergo Rayleigh instabilities and form isolated pores. Neither the pore channel radius nor the pore channel orientation are subject to control using these methods. Studies in the 1970's [38-41], examining high-temperature healing of indentation or cleavage cracks in sapphire, employed the framework developed by Nichols and Mullins to determine the ratecontrolling mass-transport mechanism, and to estimate the relevant diffusivity. Surface diffusion was identified as the rate-controlling mechanism, however, scatter in the observed normalized pore spacings ( $\mathrm{L} / \mathrm{R})$ was significant, paralleling observations on metallic systems summarized by Choy et al. [28].

Rödel and Glaeser $[13,15,16]$ initiated studies in which photolithography and ion-beam etching were used in conjunction with diffusion bonding to produce controlled-geometry, controlled-orientation pore channels in undoped sapphire. Subsequent work by Powers and Glaeser [18, 19] extended the method to ion-implanted sapphire. The etched-in channels have broad, shallow, approximately rectangular cross sections with a width and depth that can be controlled and varied. In the early stages of high-temperature annealing, the rectangular cross section rapidly becomes more equiaxed and Rayleigh instabilities become apparent along the channel sides. These instabilities eventually result in the formation of isolated pores whose spacings can be measured. By normalizing the average spacings by the radius of a circle of equivalent cross-sectional area, $\mathrm{R}_{\mathrm{eq}}$ one can assess and compare the normalized mean spacing, $\bar{L} / \mathrm{R}_{\mathrm{eq}}$, to the predictions of models for isotropic solids.

Using this microfabrication approach, pore channels were etched into the $\mathrm{c}$ and a planes of undoped sapphire [16]. At $1800^{\circ} \mathrm{C}$, channels with their axes lying in the c plane and oriented parallel to the $[1 \overline{1} 00]$ and $[11 \overline{2} 0]$ directions evolved into pores with $\bar{L} / R_{\text {eq }}$ values of 30.2-37.4 and $>200$, respectively. The values are well above those expected for an isotropic material (8.89), and differ substantially from one other. In more recent studies by Powers and Glaeser [23], a semi-infinite pore channel was rotated in $15^{\circ}$ 
increments to produce a 24-channel radial array that was etched into the $\mathrm{c}$ plane of undoped sapphire. The breakup wavelength was also highly orientation dependent, and also sensitive to the details of sample assembly. Channels oriented along the $[1 \overline{1} 00]$ had $\bar{L} / R_{\text {eq }}$ values of 45.2 and 71.6 in samples with and without an interfacial twin boundary, respectively. Channels oriented along the [1120] direction had the highest $\bar{L} / \mathrm{R}_{\text {eq }}$ values, 135 and 195, in the bonded samples with and without an intervening twin boundary, respectively, in qualitative agreement with prior observations.

This prior work explored the evolution of pore channels etched into stable surfaces. Since the cavities formed by the photolithography/ion mill etching technique are initially significantly wider $(>2 \mu \mathrm{m})$ than they are deep $(<0.5 \mu \mathrm{m})$, pores etched into stable planes are initially bound primarily by stable surfaces that may impact the characteristic wavelength of evolution. The present experiments were undertaken to examine the effect of surface stability on the evolution, and thus, pore arrays were etched into an unstable $\mathrm{m}$ plane. It was of interest to determine whether this would lead to a large decrease in $\bar{L} / R_{\text {eq }}$ values. Finally, it was of interest to characterize the cross-sectional geometry of selected channels to determine whether unusually high resistance to Rayleigh instabilities could be related to faceting.

\section{Experimental Procedures}

The general experimental procedures involved in developing microdesigned internal defect structures by lithographic methods have been described in several review papers $[17,22]$ as well as in the cited references. A brief summary is provided here with details appropriate to the present experiments, and emphasis on those procedures that differ from or augment those used previously.

\section{Materials and Sample Preparation}

Optical-grade, $99.996 \%$ pure, $1.00^{\prime \prime} \times 1.00^{\prime \prime} \times 0.020^{\prime \prime}$ sapphire crystals with the large surface within $\pm 1^{\circ}$ of $\mathbf{m}(10 \overline{10})$ were acquired from Crystal Systems, Inc.. The surface orientation was confirmed by Laue backscatter $\mathrm{x}$-ray diffraction and in-plane directions were determined concurrently. The crystals were cut into four equal pieces using a low-speed diamond saw. Fiducial marks were made on one side of the crystal pieces to facilitate proper crystal alignment during diffusion bonding.

$$
r: 7: \sim
$$


Photolithography and ion milling were performed in a Class 100 clean room. Standard cleaning procedures designed to remove organic and metallic impurities from the surface were used prior to application of an $\approx 1.8$ - $\mu \mathrm{m}$-thick layer of photoresist (Shippley 1818). Following a bake of the resist-coated substrate, a mask pattern was selectively exposed using a Canon $4 \times$ projection-mask aligner. The portion of the pattern relevant to the present results is shown in FigURE IA. Each channel is $\approx 500 \mu \mathrm{m}$ long and $\approx 3 \mu \mathrm{m}$ wide. Each radial starburst pattern contains 24 channels; the $500-\mu \mathrm{m}$-long channel is progressively rotated in $15^{\circ}$ increments to sample 12 distinct crystallographic orientations; channels at angles $\theta$ and $\theta+180^{\circ}$ are related by simple translation. The starburst pattern is repeated four times in the mask. An attempt was made to align the mask relative to the sapphire such that sets of channels would lie parallel to the [0001] and the $<11 \overline{2} 0>$ directions.

The exposed resist was developed for $1 \mathrm{~min}$ in Microposit Developer Concentrate diluted 1:1 with deionized water. The sapphire was rinsed in deionized water, dried with compressed nitrogen, and baked for $30 \mathrm{~min}$ in an air oven at $90^{\circ} \mathrm{C}$.

The sapphire with patterned photoresist was milled with $\mathrm{Ar}^{+}$ions for $45 \mathrm{~min}$ in a Veeco Microetch System ion mill. Another piece of unmilled sapphire, cut from the same original $1^{\prime \prime} \times 1^{\prime \prime}$ crystal, was set aside for diffusion bonding. The depths of the etched features $(90 \mathrm{~nm})$ were measured with an Alpha-Step IQ Surface Profiler. The $3 \mu \mathrm{m}$ feature width and $0.09 \mu \mathrm{m}$ depth combine to define a crosssectional area equivalent to that of a circle of radius $0.29 \mu \mathrm{m}$, and thus, each $500-\mu \mathrm{m}$-long channel has an initial length-to-radius ratio of $\approx 1700$, which allows the channels to be treated as semi-infinite. Crystals were recleaned with solvents, and then annealed in air for a minimum of $2 \mathrm{~h}$ at $600^{\circ} \mathrm{C}$ in a covered, highpurity alumina crucible.

The etched surface of the sapphire was brought into contact with the unmilled sapphire set aside from the same substrate, and aligned (using the fiducial marks) to assure that common in-plane directions were parallel to one another. Using substrate pairs from the same wafer avoided the bonding of substrates with different miscuts, and thereby helped minimize inadvertent misorientation during bonding. BN spacers were used to separate the external substrate surfaces from graphite foil and rams in the vacuum hot press. The assembly was diffusion bonded for $10 \mathrm{~h}$ at $1350^{\circ} \mathrm{C}$ under an applied pressure of $10 \mathrm{MPa}$.

$$
\sim: 8:
$$


Successful bonding resulted in a crystal with the etched features from the photolithography transformed into internal cavities lying on a low-angle (typically $\leq 1^{\circ}$ ) twist grain boundary as shown in FIGURE IB.

The bonded assembly was annealed at $1700^{\circ} \mathrm{C}$ in a vacuum furnace $\left(<3 \times 10^{-5}\right.$ torr; $\left.<4 \times 10^{-3} \mathrm{~Pa}\right)$. The heating and cooling rate of the furnace was $30^{\circ} \mathrm{C} / \mathrm{min}$; indicated anneal times reflect the total time at $1700^{\circ} \mathrm{C}$. Bonded crystals were annealed until the etched channels had evolved into approximately equiaxed pores that were stable to further break up. A total of 24 anneals was conducted, with the cumulative anneal time reaching $604 \mathrm{~h}$.

\section{Sample Evaluation}

Since sapphire is transparent, observation of the evolution of internal cavities by visible-light microscopy between anneals was possible, and the evolution of specific channels and features could be tracked nondestructively. The crystals were examined after bonding and after each anneal using a Zeiss visible-light microscope. The photomicrographs generated were used to determine the average pore spacing for each channel orientation. For each channel the center-to-center spacing of adjoining pores were measured and the reported values represent the average values for multiple channels of identical orientation.

Cross sections of pore channels were made using a dual-beam focused ion beam (FIB). Cross sections were made of a sample prepared with identical processing steps as for the sample in which the pore-spacing measurements were made, except it was diffusion bonded for $2 \mathrm{~h}$, etched to a depth of 0.40 $\mu \mathrm{m}$, and annealed for only $144.3 \mathrm{~h}$ at $1700^{\circ} \mathrm{C}$ so that the channels had not completely broken up into discrete pores. Before introducing the sample into the FIB, the sample was thinned substantially by conventional polishing methods to reduce the amount of material that needed to be removed by ion milling. The sample was mounted such that the plane of polish was inclined slightly relative to the plane containing the pore channels and was ground using a Planopol polishing wheel with a $15-\mu \mathrm{m}$ grit plate until some of the pore channels were partially exposed, while other channels remained completely embedded in the sapphire, but very close to the external surface. The ground surface was then polished by hand with successively finer grit diamond pastes $(15,6$, and $3 \mu \mathrm{m})$ on a nylon polishing cloth. 
After thinning the sample, optical micrographs were taken showing partially exposed and completely embedded channels. These images were used as maps to find the completely embedded channels while using FIB and SEM imaging. The locations of the embedded channels could be found relative to surface flaws such as the ends of exposed channels or scratches left from the polishing process.

Cross sections of stable pore channels oriented in the $<11 \overline{2} 0>$ direction were exposed by milling away rectangular sections of sapphire along the length of the embedded pore. Milling was performed using either a $\mathrm{Ga}^{+}$-ion beam alone or the $\mathrm{Ga}^{+}$beam enhanced with $\mathrm{I}_{2}$ gas. Imaging was performed without removing the sample from the FIB chamber using the electron beam and secondary-electron detector.

\section{Results}

The relaxation of the cross-sectional shape of pore channels has been discussed in prior studies [16, 18], and is manifested in optical micrographs by an apparent radial shrinkage of the pore channels. The initial $3 \mu \mathrm{m}$ wide by $0.09-\mu \mathrm{m}$ deep cross-section deviates significantly from an equilibrium shape, and thus there is a driving force to contract parallel to the bond plane and to expand normal to the bond plane. Similar microstructural differences and relaxation processes are evident in the current samples, and a change in contrast occurs during relaxation as the $\mathrm{m}$ surface decomposes and internal surfaces change from being perpendicular to being inclined to the incident light. For some channel orientations, facets running parallel to the channel axis may develop. The change in apparent size and contrast is illustrated in Figures $2 \mathrm{~A}$ and $2 \mathrm{~B}$, taken immediately after bonding at $1300^{\circ} \mathrm{C}$ and after an additional 20 min anneal at $1700^{\circ} \mathrm{C}$, respectively.

FIGURE 3 illustrates the morphology of a full array of channels after bonding and a subsequent 20 min anneal at $1700^{\circ} \mathrm{C}$. In some channels, periodic perturbations are already evident on the channels, as is also evident in Figure 2B. Channel-to-channel differences in contrast may reflect differences in the crosssectional structure. After five anneals at $1700^{\circ} \mathrm{C}$, totaling $8 \mathrm{~h}$, many of the channels are either completely or nearly broken up into discrete pores, as shown in Figure 4. The vertically oriented channel in this (and all prior micrographs) lies along a $<11 \overline{2} 0>$ direction, and is significantly more resistant to breakup. Whereas 
channels inclined to the $<11 \overline{2} 0>$ direction appear to break up at multiple points along the channel length within a fairly narrow time range, those oriented parallel to the $<11 \overline{2} 0>$ direction evolve by end ovulation. A time sequence of the same channel after a series of anneals of progressively increasing duration, shown in Figure 5, illustrates this behavior. Note that the two ends of the channel exhibit distinct behavior.

After a channel had evolved completely and no further breakup of pore sections was observed, the center-to-center distances between adjacent pores were measured in photomicrographs spanning the entire channel length. FIGURE 6 is a polar plot of the normalized average pore spacing, $\bar{L} / R_{\text {eq }}$, as a function of channel orientation where $\mathrm{R}_{\text {eq }}$ is the radius of an equivalent circular cross section, calculated from the area of the initial, roughly rectangular, channel cross section; the error bars represent one standard deviation. A pronounced variation in the in-plane stability is evident. Values of $\bar{L} / R_{\text {eq }}$ range from a low of 13.2 to a high of 139 for channels oriented parallel to the $\langle 11 \overline{2} 0>$ direction; the standard deviations are on the order of $35 \%$ of $\bar{L} / R_{\text {eq }}$.

Twenty-four anneals were used to examine the temporal evolution of the channels. As channels of particular orientations neared breakup, anneal durations were adjusted to try to narrowly define the cumulative period of annealing for breakup. However, since in situ measurements were not possible, it is only possible to indicate a time window in which the breakup was completed. FIGURE 7A presents a polar plot of the range of breakup times for each orientation. Despite the resulting uncertainty, trends in the breakup times parallel those in the breakup wavelength. The average breakup times for the eight channels of each orientation ranged from 2 to $468 \mathrm{~h}$, and as a result, a log scale is used for the times in FIgURE 7A. For most orientations, average breakup times were less than or equal to $5 \mathrm{~h}$. Channels oriented parallel to [0001], completed breakup in times ranging from 2 to $20 \mathrm{~h}$, and on average in $10 \mathrm{~h}$. The most pronounced stability was exhibited by channels oriented parallel to a $<11 \overline{20}>$ direction, which required between 228 to $604 \mathrm{~h}$ for complete breakup, and on average $468 \mathrm{~h}$. Simple models of the kinetics suggest that the evolution times should be proportional to the square of the perturbation wavelength [31]. FIGURE 7B presents a polar plot of the breakup times normalized by the square of the mean pore spacing, $\bar{L}^{2}$. Note that the time scale is now linear. 
The enhanced stability of channels oriented along a $<11 \overline{2} 0>$ direction permitted and prompted an examination of the cross-sectional shape of these pore channels. An SEM image of a cross section is provided in Figure 8; the channel is bounded by facets, and appears oblong rather than equiaxed. Due to the geometry of the dual-beam FIB and the limitations on the tilt range of the stage, SEM images made in situ, immediately after FIB milling, could not be made with the electron beam normal to the cross section. Instead, the cross section was imaged with the e-beam at a tilt of $30^{\circ}, 38^{\circ}$, or $60^{\circ}$ from normal. When the image is corrected for this effect, the facets bounding the channel can be properly identified and are shown to be $\mathbf{c}, \mathbf{r}$, and $\mathbf{s}$ facets. These facets are components of the Wulff shape [12, 36, 37]. However, even when the image is corrected for sample tilt, the cross-sectional shape is not as equiaxed as would be expected for full 2-D equilibration.

\section{Discussion}

The onset of axial instability is preceded by or concurrent with a relaxation of the cross-sectional shape of the lithographically introduced pores, as is evident in comparing Figures 2A and 2B, and is qualitatively consistent with prior observations for pores etched into the $\mathbf{c}(0001)$ surface of sapphire. The initial cross-sectional shape, if approximated as rectangular, involves a width-to-depth ratio of 3.0 to 0.09 or $\approx 33$. The extreme deviation from an equilibrium aspect ratio and shape drives rapid transport transverse to the channel axis. In sapphire, on the length scale of the channel dimensions, surface diffusion is expected to be the rate-limiting transport process. Surface-diffusion-limited 2-D relaxation has been modeled for various initial geometries [10]. Assuming that the cross section remains rectangular, the equilibrium crosssectional shape is square, and an average reported value of the surface diffusivity of alumina at $1700^{\circ} \mathrm{C}$ applies, one would expect an anneal of roughly $12 \mathrm{~min}$ would be necessary to reduce the width-to-diameter ratio to 1.01 (which would be experimentally indistinguishable from the equilibrium shape). The modeling makes numerous simplifying assumptions, and thus, it is the order of magnitude that is relevant. Since for some orientations channel breakup has already initiated within the first 20 min anneal, the calculation merely suggests that axial instabilities may initiate prior to achieving an equiaxed cross section, and that the 
actual channel break-up behavior is more complex than that described by existing models. Efforts to model axial instabilities in cases where the cross-sectional shapes differ from the 2-D "equilibrium" shape are in progress. In the present case, the development of new facets can also be expected as part of the shape relaxation process. Notable in this regard is the possibility for channels oriented parallel to a $<11 \overline{2} 0>$ direction and the [0001] direction to become fully bounded by facets. In the context of EQN. (1), this would lead to a strong stabilization to axial instability. Flaws in the channels due to processing errors and the large "perturbations" that occur at the ends of the channels are then expected to play a more critical role in the breakup, consistent with observations.

It is evident from Figure 4 that the evolution wavelengths and times can vary considerably as the channel orientation and the nature of the bounding surfaces are changed. FIGURE 5 indicates that the mechanism leading to breakup can also be affected by the channel orientation. For channels that cannot be bounded by facets that extend parallel to the channel axis, periodic oscillations develop along the channel length, qualitatively paralleling the behavior of fluid jets and of pore channels developed during crack healing. It is interesting to note that in this and prior studies, differences between the initial perturbation wavelengths and final pore spacings have been observed [18], indicating that the initially dominant perturbation need not prevail throughout the channel evolution. Breakup at various points along the channel, and associated end retraction when local pinchoff of the channel occurs may also contribute to these differences. As discussed, when stable facets bound the channel, the "shedding" of isolated pores from the ends of the channels becomes the dominant process. This evolution mode has been modeled for isotropic materials [42], but to our knowledge, parallel behavior in anisotropic materials has not. Anisotropy-induced shifts in the spacing of pores are to be expected, and the behavior of channels oriented parallel to a $<11 \overline{2} 0>$ direction provide direct supporting experimental evidence. As is the case with periodic perturbations, the adjustment in the energetics will have concomitant effects on the time per ovulation event.

When the distribution in $\bar{L} / R_{\text {eq }}$ values is examined, FIGURE 6, two features of interest emerge. The $\bar{L} / R_{\text {eq }}$ values for all sampled orientations exceed 8.89 , the value predicted for isotropic systems undergoing surface-diffusion-controlled evolution. Even the minimum measured center-to-center pore 
spacing within each channel exceeds $8.89 \mathrm{R}_{\text {eq }}$. For most channel orientations, and all that are unique to the m-plane substrates, the measured values of $\bar{L} / R_{\text {eq }}$ are well below those characteristic of channels etched into the stable c plane [23]. Thus, the shift in the $\bar{L} / \mathrm{R}_{\mathrm{eq}}$ values is in a direction consistent with the relative stabilities of the $\mathrm{m}$ and $\mathrm{c}$ planes that dominate the surface crystallography of the etched-in pores. The presence of a stable c-plane facet in channels etched into the c-plane may help stabilize the channels relative to those etched into the $\mathrm{m}$-plane. In the $\mathrm{m}$-plane substrates, most $\bar{L} / \mathrm{R}_{\text {eq }}$ values exceed 8.89 by a sufficiently modest amount that explanations for the positive shift involving wave-wave interactions and the influence of pre-existent perturbations ("noise") [28] could be invoked. A prima facie assessment of EQN. (1) would invite an interpretation that noise is responsible for the shift in $\lambda_{\max }$ to values $>8.89 \mathrm{R}$. There are, however, several considerations that suggest that the shift has other causes, specifically SEA. The strong directional dependence of the shift observed in this and prior c-plane studies and the difference in the behavior of these crystallographically distinct samples seems inconsistent with noise being solely responsible. The shifts observed in prior studies in c-plane substrates [23], and for channels oriented parallel to $\mathrm{a}<11 \overline{2} 0>$ direction in the $\mathrm{m}$-plane substrates also seem too large to be rationalized in this way, particularly since the initial artificially introduced patterns are less "noisy" than typical natural systems. Finally, experiments in which the growth of prescribed finite-amplitude perturbations was studied [20] showed significant shifts in $\bar{L} / \mathrm{R}_{\text {eq }}$ values in situations where the scatter in pore spacings, an indicator of "noise", was minimal.

The behavioral differences observed between $\mathrm{m}$ and $\mathrm{c}$ planes in the Rayleigh-instability experiments are also consistent with other studies on the morphological evolution of $\mathbf{m}$ and $\mathrm{c}$ surfaces during annealing [33-35]. Based on the findings of Heffelfinger et al. one expects that the $\mathrm{m}$ plane will rapidly undergo decomposition into $\mathbf{r}$ and $\mathbf{s}$ planes at a much smaller length scale than $\lambda_{\text {min }}$ for pore channels of the size examined in this study; vicinal $c(0001)$ planes develop terrace-ledge structures. The initial crystallographic differences in the character of the bounding surfaces for these two types of channels lead to different surface topographies, which in turn affect evolution rates. The "rough" hill-and-valley structure appears to result in much more rapid shape relaxation kinetics [11], reducing the extent of any residual bounding segments parallel to the original $\mathrm{m}$ plane. As a result of the significantly more sluggish

$$
r: 14: \sim
$$


reduction in the extent of $\mathrm{c}$-plane segments, channels etched into the two crystal surfaces may undergo differing degrees of cross-sectional relaxation before the onset of substantial axial instability. This can also lead to distinct behavior for channels of identical axial orientation etched into the $\mathbf{m}$ and $\mathbf{c}$ planes. For example, although channels oriented parallel to $\mathrm{a}<11 \overline{2} 0>$ direction in the $\mathrm{m}$ plane and channels etched into the $\mathrm{c}$ plane also oriented along a $<11 \overline{2} 0>$ direction both had the greatest resistance to breakup and the highest $\bar{L} / \mathrm{R}_{\text {eq }}$ values, the $\bar{L} / \mathrm{R}_{\text {eq }}$ values differ by almost $50 \%$. It is possible that the two cross-sectional geometries are not the same at the onset of substantial axial instability. Rödel and Glaeser [16] previously reported similar differences in the behavior of channels oriented along the same crystallographic direction but etched into and initially bounded by differing planes.

The origin of the enhanced stability of channels oriented along a $<11 \overline{2} 0>$ direction was examined by sectioning pore channels and other (even larger) pattern markers such as the arrows evident in FIGURE I. When such cross sections are corrected for tilt angle between the electron beam and the cross section, the orientations of the surfaces with respect to the known in-plane directions can be determined. Assessments of several channel cross sections indicate that the bounding stable facets are $\mathbf{c}, \mathbf{r}$, and $\mathbf{s}$ facets. Measurements of the equilibrium (Wulff shape) at both $1600^{\circ} \mathrm{C}$ and $1800^{\circ} \mathrm{C}$ have been performed $[12,36]$, and the Wulff theorem suggests that the spacings between parallel sets of the $\mathbf{c}, \mathbf{r}$, and $\mathbf{s}$ facets should be in the ratio 1.0:0.855:0.947 at $1600^{\circ} \mathrm{C}$, and 1.0:0.95:1.04 at $1800^{\circ} \mathrm{C}$ when a particle is viewed along a $<11 \overline{2} 0>$ direction. Assuming that the channels would adopt a similar configuration, and continuous variations of these ratios with temperature, at $1700^{\circ} \mathrm{C}$ the ratios of facet spacings should lie between 0.855 and 1.04 . Features with initial $6 \mu \mathrm{m}$ by $0.4 \mu \mathrm{m}$, or $10 \mu \mathrm{m}$ by $0.4 \mu \mathrm{m}$ cross-sections, and initial width-to-depth ratios of 15 and 24 , respectively, were sectioned using the FIB. After annealing for $144.3 \mathrm{~h}$ at $1700^{\circ} \mathrm{C}$ the average measured $\mathrm{c}, \mathbf{r}$, and $\mathbf{s}$ facet spacings are in the ratio 1.0:0.65:1.05 and 1.0:0.77:1.10, for the smaller and larger cross-sections, respectively. Although significant shape relaxation has occurred, it is not complete. For rectangular cross sections having equivalent cross-sectional areas but relaxing to a square cross section an anneal of $144.3 \mathrm{~h}$ would be expected to produce aspect ratios of $<1.0001$ in both cases, and an aspect ratio of 1.01 is expected after $15 \mathrm{~h}$ and $42 \mathrm{~h}$ for the smaller and larger channels [10], respectively. Qualitatively, evolution appears to be more sluggish than would be expected for surface-diffusion-controlled evolution. Prior studies of the

$$
r: 15: \sim
$$


shape relaxation of pores of identical geometry but etched into $\mathrm{c}$ - and $\mathrm{m}$-plane sapphire have shown pronounced relaxation rate differences [11]. Mechanistically, in the absence of time-independent sources of ledges on the bounding facets (such as those evident in FIGURE 8), facet migration and the development of axial perturbations may require the nucleation and subsequent lateral growth of critical-size atomic-height patches and cavities on facets moving radially inward and outward [43], respectively. As discussed by Mullins and Rohrer, this can introduce a nucleation energy barrier that can be difficult to overcome. Under such circumstances, extremely sluggish evolution would occur. For systems undergoing Rayleigh instabilities, evolution would be dominated by perturbations arising from processing errors or associated with the ends of the channel. This is consistent with observations.

Channels oriented along the $\langle 11 \overline{2} 0>$ and [0001] directions are the only ones that can be fully bounded by facets that are part of the equilibrium shape. As illustrated, channels oriented along $<11 \overline{2} 0>$ are fully bounded by $\mathbf{c}, \mathbf{r}$, and $\mathbf{s}$ facets, have the highest value of $\bar{L} / \mathrm{R}_{\text {eq }}$, and are the most resistant to breakup. Channels oriented along [0001] within the $\mathrm{m}$ plane can in principle develop six bounding a facets along the channel perimeter, and channels of this orientation have the second highest value of $\bar{L} / \mathrm{R}_{\mathrm{eq}}$. The a facets have either one of the higher energies $\left(1600^{\circ} \mathrm{C}\right)$ or the highest energy $\left(1800^{\circ} \mathrm{C}\right)$ of the stable planes, and the equilibrium form, when viewed along [0001] is not fully bounded by a facets [12]. This may contribute to the more modest stabilization of these channels. Channels in several other directions may also be stabilized by partially faceted cross sections if the $\mathrm{m}$ plane and the $\mathbf{r}, \mathbf{s}$ or $\mathbf{p}$ planes have a zone axis that corresponds to the axis of an etched-in channel. A detailed analysis of this, and a re-evaluation of prior work in c-plane sapphire attentive to this issue are in progress.

In the model anisotropic system originally modeled by Cahn [7] transverse isotropy in the surface energy preserves a circular cross section in the plane normal to the cylinder axis. By limiting anisotropy to a plane parallel to the cylinder axis, the determination of both $\lambda_{\min }$ and $\lambda_{\max }$ are simplified, and both values are affected by the orientation dependence of the surface energy. The kinetic analysis of Stölken and Glaeser [29] assumes that the evolution is surface-diffusion limited. An important outcome of this formulation is that the time required to grow a fixed initial amplitude perturbation to the point of breakup is predicted to be proportional to $\mathrm{R}^{4} \lambda_{\text {max }}^{2} / \mathrm{D}_{s}$ where $\mathrm{D}_{s}$ is the surface diffusivity. Avoiding the breakup of 
fine-scale rods or wires requires that low processing temperatures with low $D_{s}$ be used, or that $\lambda_{\text {max }}$ be increased by exploiting SEA and possibly using surface-active impurities to enhance the stability of bounding surfaces. Although wide variations in the orientation-dependent values of $\lambda_{\max }$ and the breakup time have been documented in this study and in prior work, the model suggests that if the breakup times are normalized by $\lambda_{\max }^{2}$, the variations will be substantially reduced. Thus, in principle, the behavior of channels with widely varying $\lambda_{\text {max }}$ can be consolidated by this normalization. FIGURE 7 provides an indication of the extent to which such a consolidation is successful. The most serious outlier is the data for channels oriented parallel to a $<11 \overline{2} 0>$ direction. Since these evolve by end ovulation rather than nearsimultaneous pinchoff at minima along the channel, this deviation is not unexpected.

\section{Summary and Conclusions}

Arrays of controlled-orientation semi-infinite pore channels were introduced into undoped $\mathrm{m}$ plane sapphire substrates using microfabrication techniques and ion-beam etching and subsequently internalized by solid-state diffusion bonding. The breakup of these artificially generated intergranular pore channels into discrete particles via Rayleigh instabilities during a series of anneals at $1700^{\circ} \mathrm{C}$ was monitored. In all cases, channels broke up with a characteristic wavelength larger than that expected for a material with isotropic surface energy (8.89R). We attribute this to stabilization effects due to surface energy anisotropy. The breakup wavelength and the times required for complete breakup varied with in-plane channel orientation. For most orientations, the normalized average pore spacing, $\bar{L} / \mathrm{R}_{\mathrm{eq}}$, was in the range of 13.2 to 25, and complete breakup occurred within 2-10 h; these normalized pore spacings are less than those for channels introduced into the stable $\mathrm{c}$ plane of sapphire. To first order, the anneal times for complete breakup scale with $\left(\bar{L} / \mathrm{R}_{\text {eq }}\right)^{2}$. Channels oriented along a $<11 \overline{2} 0>$ direction had an $\bar{L} / \mathrm{R}_{\text {eq }}$ value of $\approx 139$, and required $468 \mathrm{~h}$ for complete breakup. Thus, the potential for significant morphological stabilization exists. Cross-sectional analysis of channels oriented along a $<11 \overline{2} 0>$ direction revealed them to be completely bounded by stable $\mathbf{c}, \mathbf{r}$, and $\mathbf{s}$ facets, facets that are part of the equilibrium or Wulff shape of undoped sapphire. 


\section{Acknowledgements}

This research was supported by the Director, Office of Science, Office of Basic Energy Sciences, Division of Materials Science and Engineering, of the U.S. Department of Energy under Contract No.

DE-AC03-76SF00098. We are grateful to the staff of the Microfabrication Laboratory at the University of California for their assistance. Melissa Santala acknowledges support in the form of a Eugene Cota-Robles Fellowship and a National Science Foundation Graduate Fellowship. Access to the National Center for Electron Microscopy at the Lawrence Berkeley National Laboratory and the assistance of Dr. Andrew Minor are appreciated. 


\section{REFERENCES:}

1. M.T. Plateau, Philosophical Magazine S4 12 (1856) 286-97.

2. L. Rayleigh, Proceedings of the London Mathematical Society 10 (1879) 4-13.

3. F.A. Nichols, W.W. Mullins, Trans. A.I.M.E. 233 (1965) 1840-48.

4. F.A. Nichols, W.W. Mullins, J. Appl. Phys. 36 (1965) 1826-35.

5. $\quad$ G. Wulff, Z. Krist. 34 (1901) 449-530.

6. C. Herring, Physical Review 82 (1951) 87-93.

7. J.W. Cahn, Scr. Metall. (USA) 13 (1979) 1069-71.

8. C. Herring, J. Appl. Phys. 21 (1950) 301-03.

9. M. Kitayama, A.M. Glaeser, Key Engin. Mater. 159-160 (1999) 193-204.

10. M. Kitayama, T. Narushima, W.C. Carter, R.M. Cannon, A.M. Glaeser, J. Am. Ceram. Soc. (USA) 83 (2000) 2561-71.

11. M. Kitayama, T. Narushima, A.M. Glaeser, J. Am. Ceram. Soc. (USA) 83 (2000) 2572-83.

12. M. Kitayama, A.M. Glaeser, J. Am. Ceram. Soc. (USA) 85 (2002) 611-22.

13. J. Rödel, A.M. Glaeser, J. Am. Ceram. Soc. (USA) 70 (1987) C172-5.

14. J. Rödel, A.M. Glaeser, Mater. Lett. 6 (1988) 351-55.

15. J. Rödel, A.M. Glaeser, J. Am. Ceram. Soc. 73 (1990) 592-601.

16. J. Rödel, A.M. Glaeser, in: C.A. Handwerker, J.E. Blendell, W.A. Kaysser (Eds.), Sintering of Advanced Ceramics, Westerville, OH, 1990, pp. 243-57.

17. J. Rödel, A.M. Glaeser, Yogyo Kyokai Shi 99 (1991) 251-65.

18. J.D. Powers, A.M. Glaeser, J. Am. Ceram. Soc. (USA) 75 (1992) 2547-58.

19. J.D. Powers, A.M. Glaeser, J. Am. Ceram. Soc. 76 (1993) 2225-34.

20. L. Kulinsky, J.D. Powers, A.M. Glaeser, Acta Mater. 44 (1996) 4115-30.

21. M. Kitayama, A.M. Glaeser, J. Mater. Synth. Proc. 6 (1998) 161-7.

22. M. Kitayama, J.D. Powers, L. Kulinsky, A.M. Glaeser, J. Eur. Ceram. Soc. 19 (1999) 2191-209.

23. J.D. Powers, A.M. Glaeser, J. Am. Ceram. Soc. (USA) 83 (2000) 2297-304.

24. T. Narushima, A.M. Glaeser, J. Am. Ceram. Soc. (USA) 84 (2001) 921-8.

25. A.M. Glaeser, Interface Science 9 (2001) 65-82.

26. W.C. Carter, A.M. Glaeser, Mater. Sci. Eng. 89 (1987) L41-L45.

27. R.F. Sekerka, T.F. Marinis, in: H.I. Aaronson, D.E. Laughlin, R.F. Sekerka, C.M. Wayman (Eds.), SOLID $\rightarrow$ SOLID PHASE TRANSFORMATIONS, New York, 1982, pp. 67-84.

28. J.-H. Choy, S.A. Hackney, J.K. Lee, J. Appl. Phys. 77 (1995) 5647-54.

29. J.S. Stölken, A.M. Glaeser, Scr. Metal. Mater. 27 (1992) 449-54.

30. K.F. Gurski, G.B. McFadden, Proc. R. Soc. Lond. A, Math. Phys. Sci. (UK) 459 (2003) $2575-$ 2598.

31. A.M. Glaeser, in: K. Koumoto, L.M. Sheppard, H. Matsubara (Eds.), Mass and Charge Transport in Ceramics, Westerville, 1996, pp. 117-36.

32. D.W. Susnitzky, C.B. Carter, J. Am. Ceram. Soc. (USA) 75 (1992) 2463-78.

33. J.R. Heffelfinger, M.W. Bench, C.B. Carter, Surface Science 343 (1995) L1161-6.

34. J.R. Heffelfinger, M.W. Bench, C.B. Carter, Surface Science 370 (1997) L168-72.

35. J.R. Heffelfinger, C.B. Carter, Surface Science 389 (1997) 188-200.

36. M. Kitayama, The Wulff Shape of Doped and Undoped Sapphire, Berkeley, University of California, 1996.

37. J.-H. Choi, D.-Y. Kim, B.J. Hockey, S.M. Wiederhorn, C.A. Handwerker, J.E. Blendell, W.C. Carter, A.R. Roosen, J. Am. Ceram. Soc. (USA) 80 (1997) 62-8. 
38. C.F. Yen, R.L. Coble, J. Am. Ceram. Soc. (USA) 55 (1972) 507-9.

39. A.G. Evans, E.A. Charles, Acta Metall. (USA) 25 (1977) 919-27.

40. T.K. Gupta, J. Am. Ceram. Soc. (USA) 61 (1978) 191-5.

41. T.K. Gupta, in: W.D. Kingery (Ed.), Advances in Ceramics, Columbus, 1984, pp. 750-66.

42. F.A. Nichols, J. Mater. Sci. (UK) 11 (1976) 1077-82.

43. W.W. Mullins, G.S. Rohrer, J. Am. Ceram. Soc. (USA) 83 (2000) 214-16. 


\section{Figure Captions}

FIgURE I a) Schematic illustration of the mask pattern used to generate the radial pore-channel array, and b) an internalized array in $\mathbf{m}$-plane sapphire after bonding, but prior to any annealing at $1700^{\circ} \mathrm{C}$. The diameter of the channel array is $\approx 1050 \mu \mathrm{m}$.

FIGURE 2 Higher magnification optical micrographs of a) an internalized pore channel array after bonding at $1300^{\circ} \mathrm{C}$, and b) an array after a 20 -min anneal at $1700^{\circ} \mathrm{C}$. Note the change in contrast, and the onset of breakup in some channels.

Figure 3 Optical micrographs of a) an internalized pore channel array after bonding at $1300^{\circ} \mathrm{C}$, and b) an array after a $20 \mathrm{~min}$ anneal at $1700^{\circ} \mathrm{C}$. The breakup of many channels has initiated. The vertical channels are aligned along a $<11 \overline{2} 0\rangle$ direction.

Figure 4 Optical micrograph of an internal pore channel array after $8 \mathrm{~h}$ cumulative annealing at $1700^{\circ} \mathrm{C}$. The vertical channels are aligned along a $<11 \overline{2} 0>$ direction.

FigURE 5 Sequence of optical micrographs showing a particular pore channel aligned along a $<11 \overline{20}>$ direction after a) 16 h, b) 228 h, c) 300 h, d) 444 h, and e) $604 \mathrm{~h}$ of annealing at $1700^{\circ} \mathrm{C}$.

FIGURE 6 A polar plot of the normalized average pore spacing, $\bar{L} / \mathrm{R}_{\mathrm{eq}}$, as a function of channel orientation. The outermost and innermost points along each direction represent the mean plus and minus one standard deviation, respectivley. For comparison, the behavior expected for an isotropic system evolving by surface diffusion is indicated.

Figure $7 \quad$ a) Polar plot of the maximum and minimum times for complete breakup with time on a log scale. b) Polar plot of maximum and minimum breakup times normalized by the square of the mean pore spacing; note the normalized time scale is linear.

Figure 8 Scanning electron micrograph of a cross-section taken perpendicular a $<11 \overline{2} 0>$ direction. Stable channels and features with long axes aligned parallel to a $<11 \overline{2} 0>$ direction develop bounding $\mathbf{c}, \mathbf{r}$, and $\mathbf{s}$ facets, as indicated. 


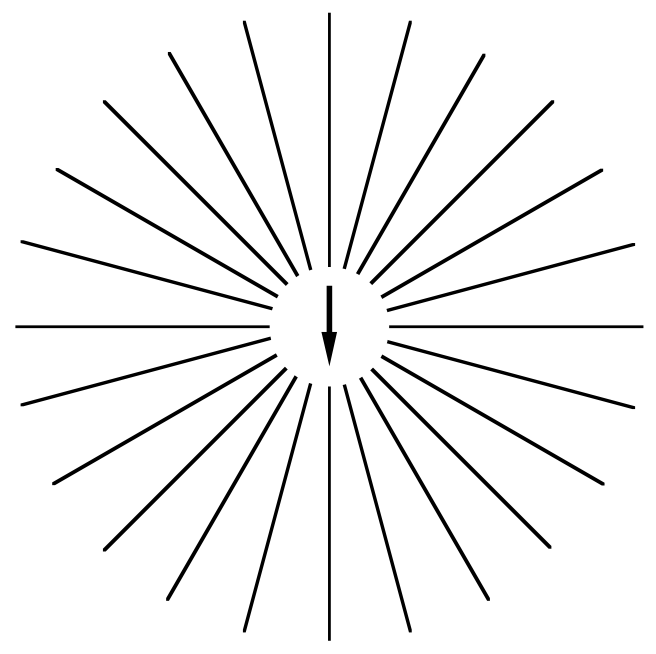

(a)

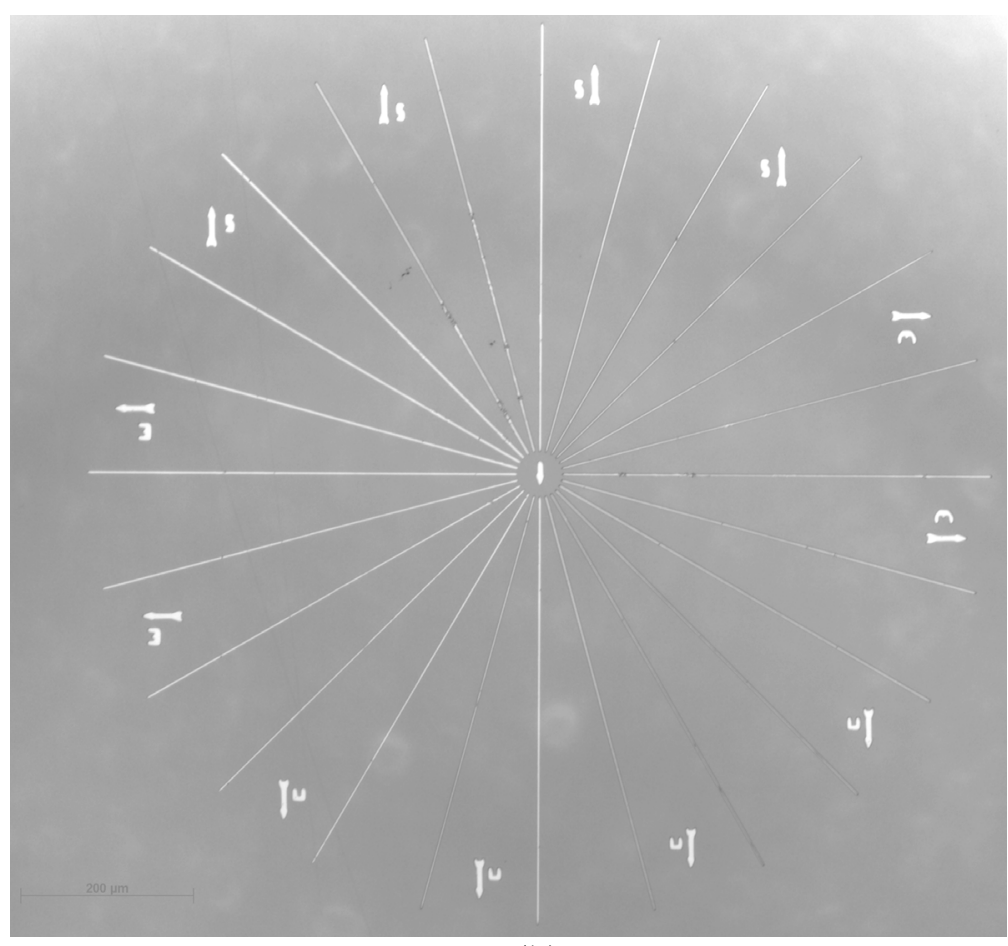

(b)

Figure I a) Schematic illustration of the mask pattern used to generate the radial pore-channel array, and b) an internalized array in $\mathrm{m}$-plane sapphire after bonding, but prior to any annealing at $1700^{\circ} \mathrm{C}$. The diameter of the channel array is $\approx 1050 \mu \mathrm{m}$. 


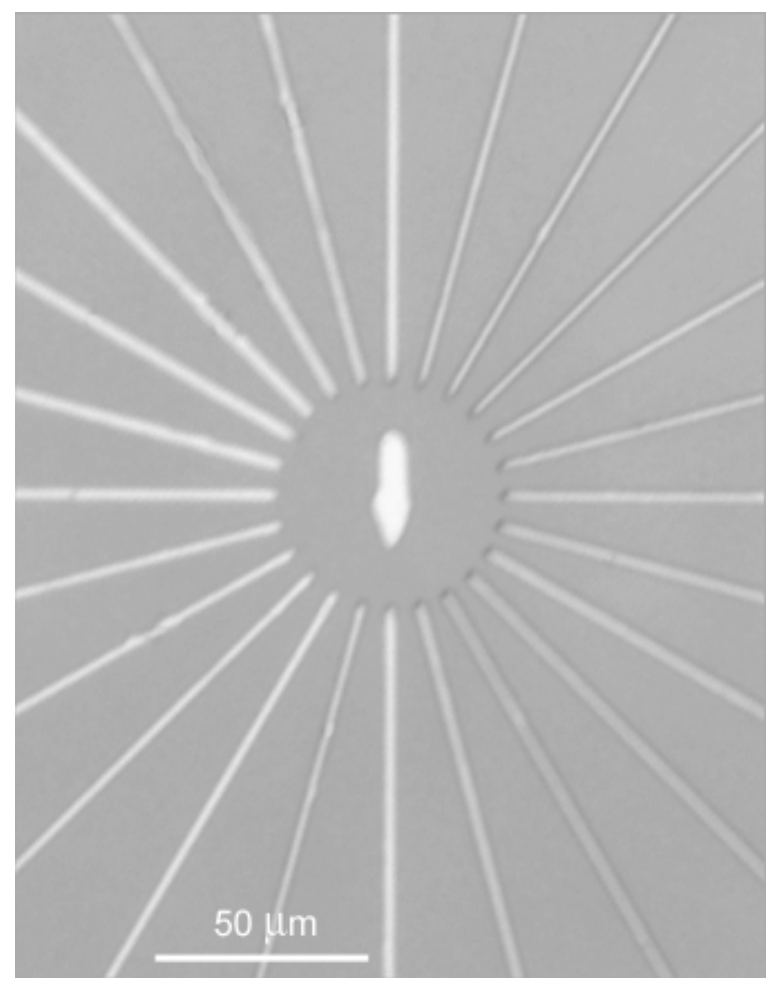

(a)

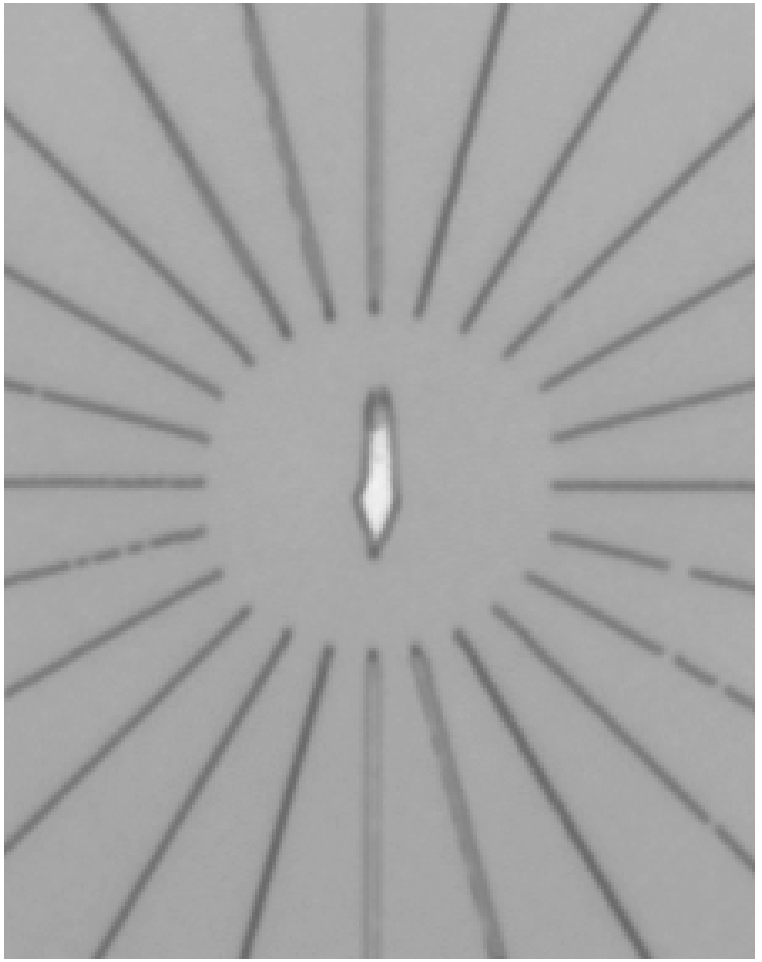

(b)

Figure 2 Higher magnification optical micrographs of a) an internalized pore channel array after bonding at $1300^{\circ} \mathrm{C}$, and b) an array after a 20 -min anneal at $1700^{\circ} \mathrm{C}$. Note the change in contrast, and the onset of breakup in some channels. 

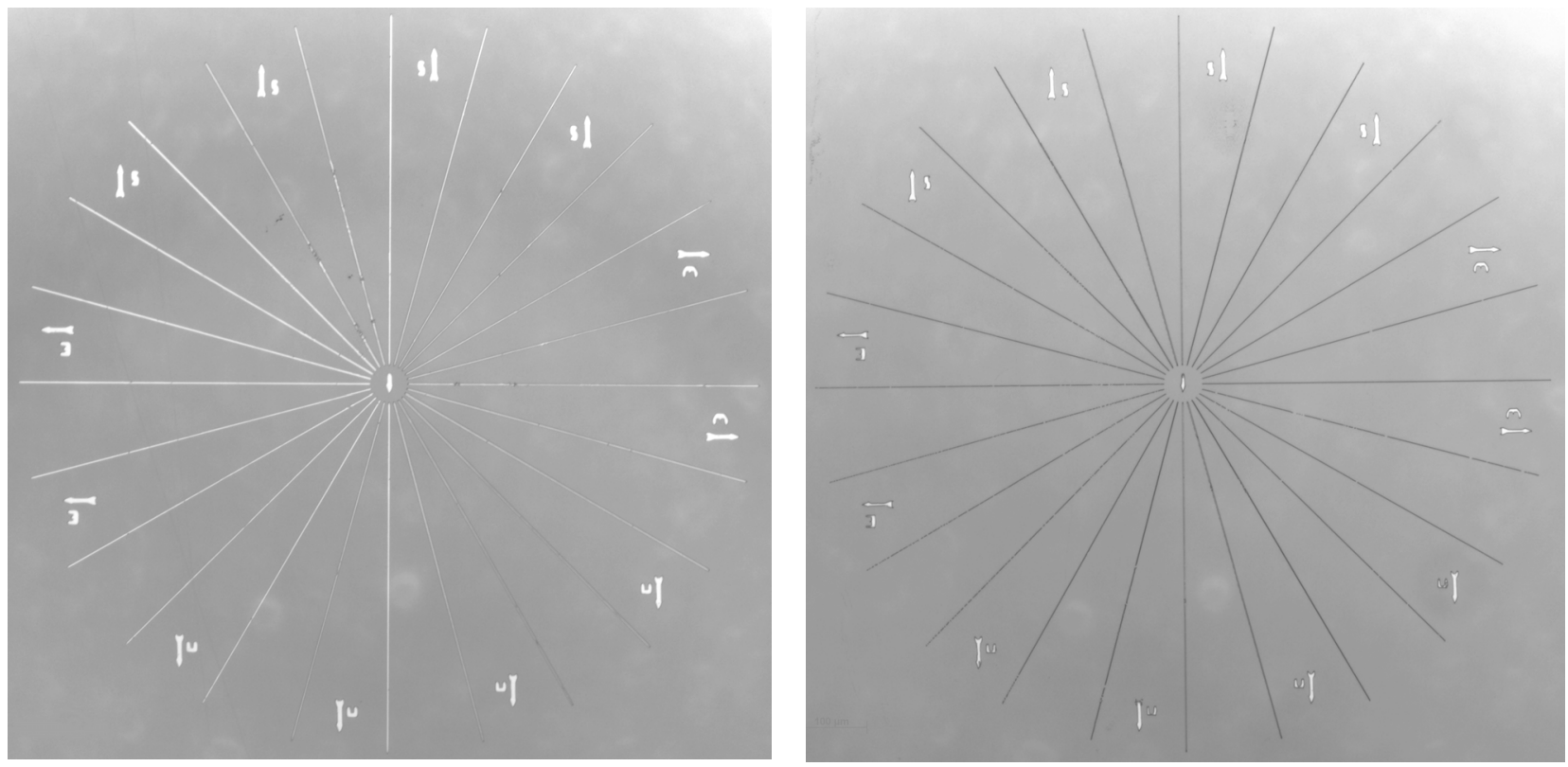

Figure 3 Optical micrographs of a) an internalized pore channel array after bonding at $1300^{\circ} \mathrm{C}$, and b) an array after a $20 \mathrm{~min}$ anneal at $1700^{\circ} \mathrm{C}$. The vertical channels are aligned along a $<11 \overline{2} 0>$ direction. 


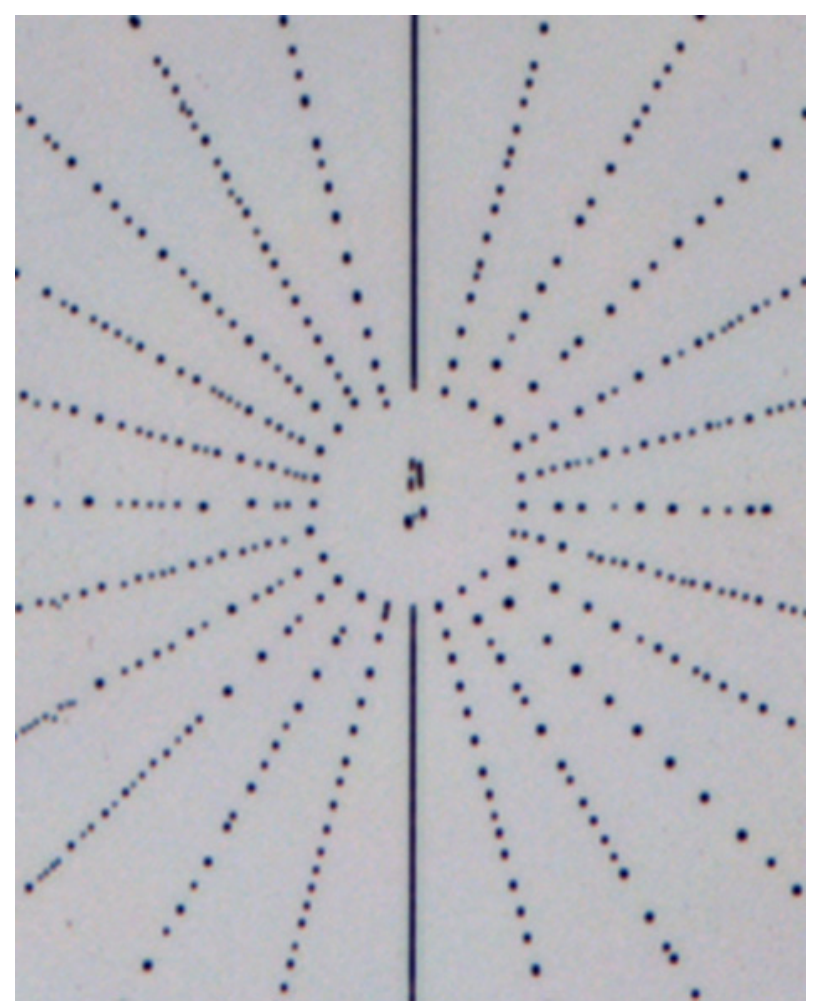

Figure 4 Optical micrograph of an internal pore channel array after $8 \mathrm{~h}$ cumulative annealing at $1700^{\circ} \mathrm{C}$. The vertical channels are aligned along a $<11 \overline{2} 0>$ direction. 


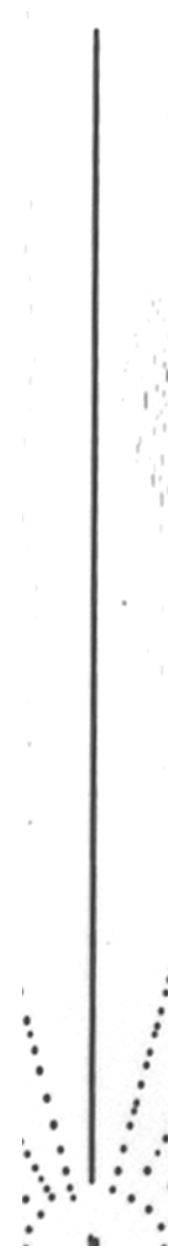

(a)

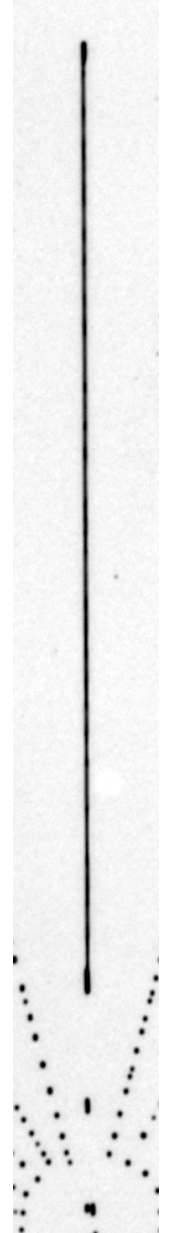

(b)

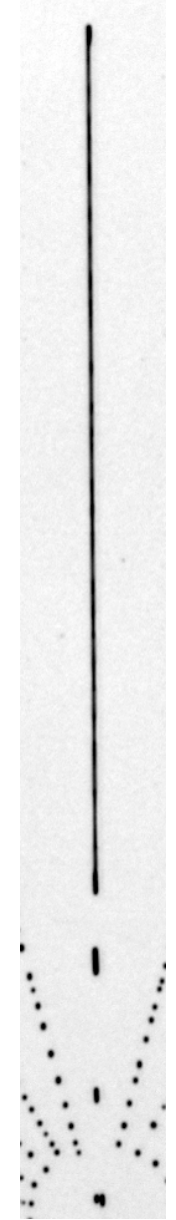

(c)

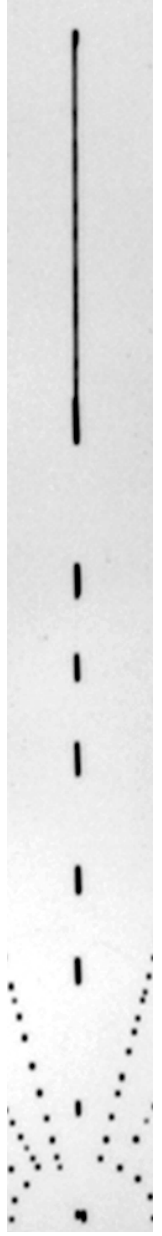

(d)

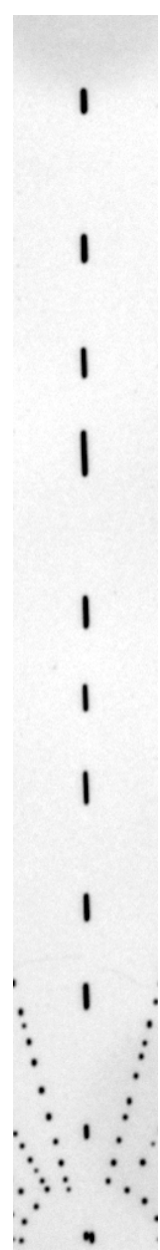

(e)

Figure 5 Sequence of optical micrographs showing a particular pore channel aligned along a $<11 \overline{2} 0>$ direction after a) $16 \mathrm{~h}, \mathrm{~b}) 228 \mathrm{~h}$, c) $300 \mathrm{~h}$, d) $444 \mathrm{~h}$, and e) $604 \mathrm{~h}$ of annealing at $1700^{\circ} \mathrm{C}$. 


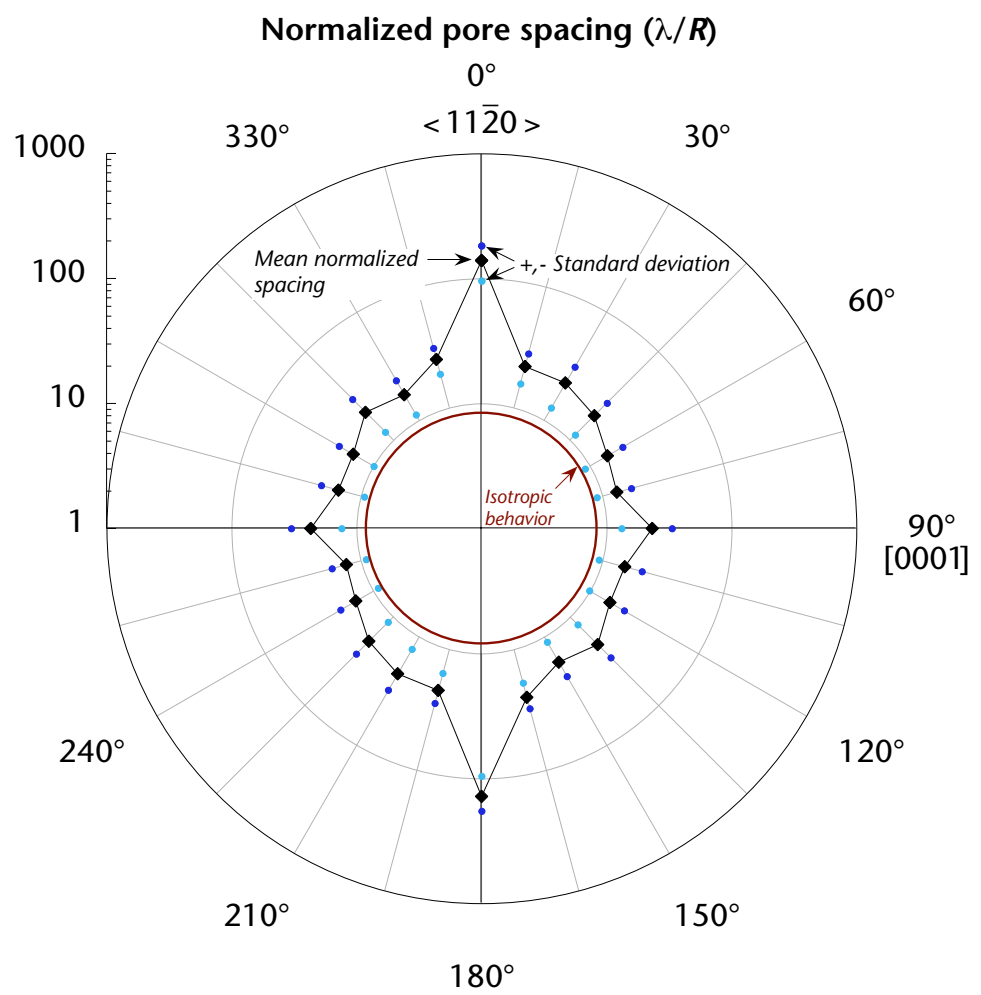

Figure 6 A polar plot of the normalized average pore spacing, $\bar{L} / \mathrm{R}_{\text {eq }}$, as a function of channel orientation. The outermost and innermost points along each direction represent the mean plus and minus one standard deviation, respectively. For comparison, the behavior expected for an isotropic system evolving by surface diffusion is indicated. 
Time to complete break up (hours)

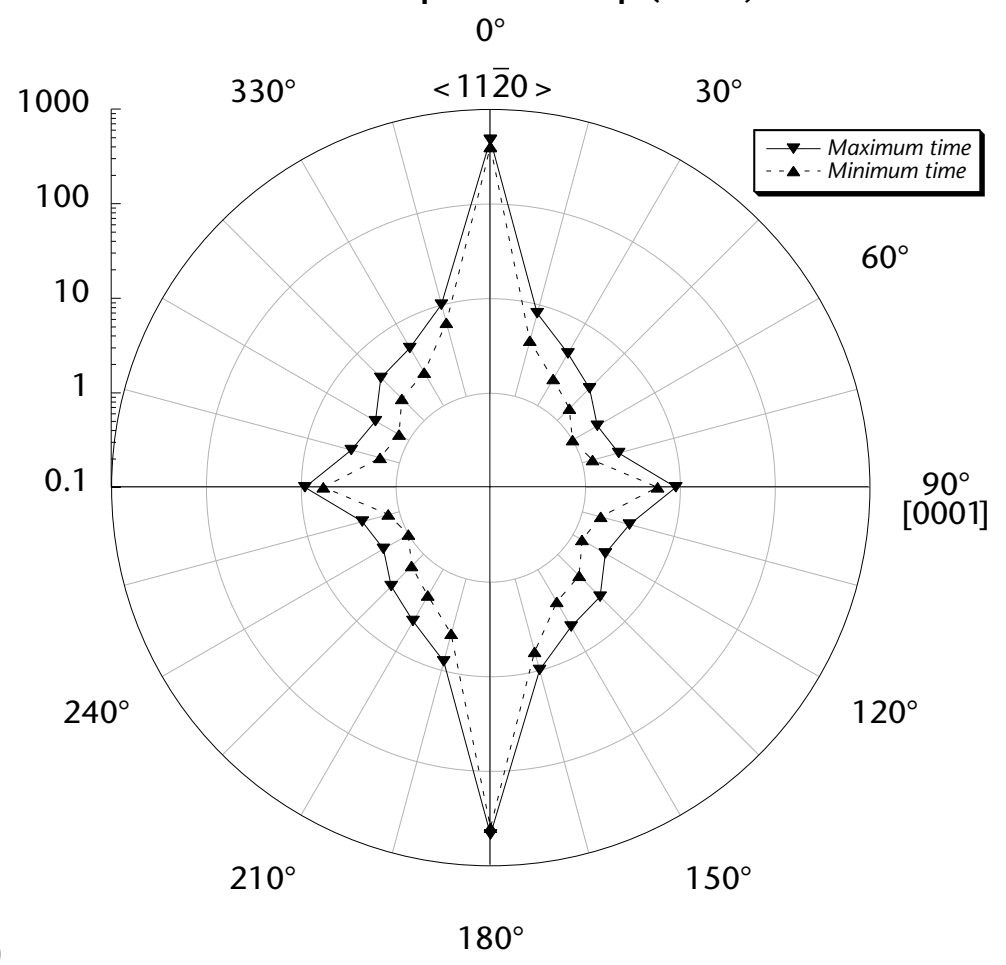

a)

\section{Normalized time to complete break up Anneal time $/ \bar{L}^{2}$}

$0^{\circ}$

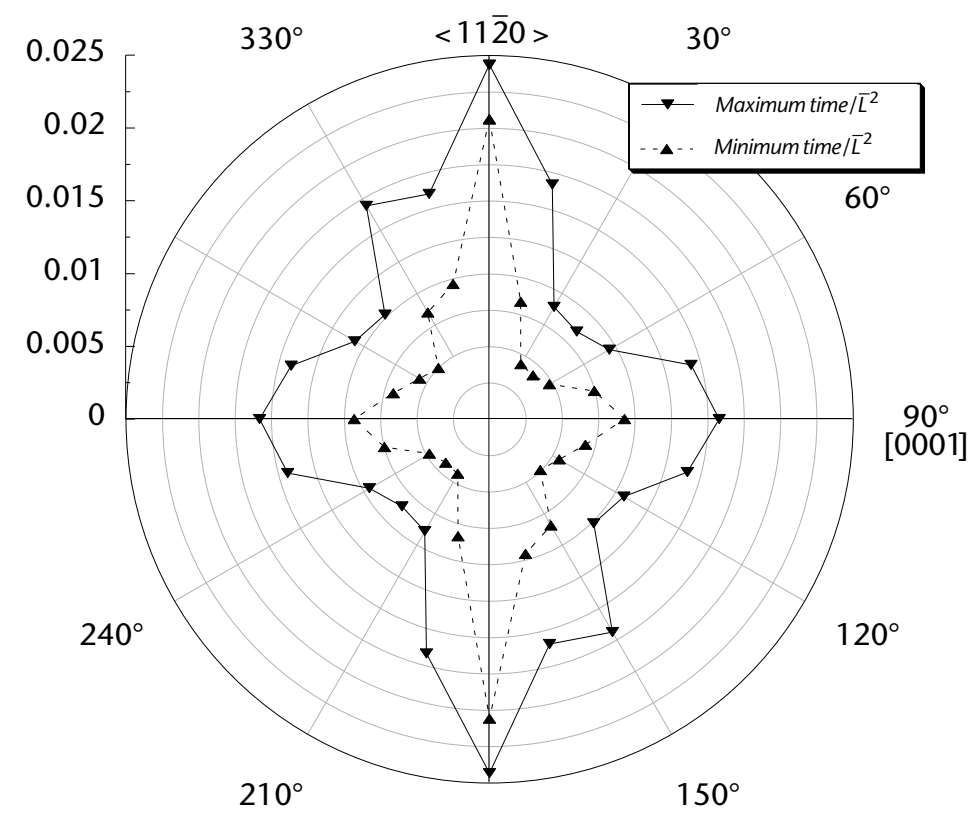

b)

$180^{\circ}$

Figure 7 a) Polar plot of the maximum and minimum times for complete breakup with time on a log scale. b) Polar plot of maximum and minimum breakup times normalized by the square of the mean pore spacing; note the normalized time scale is linear. 


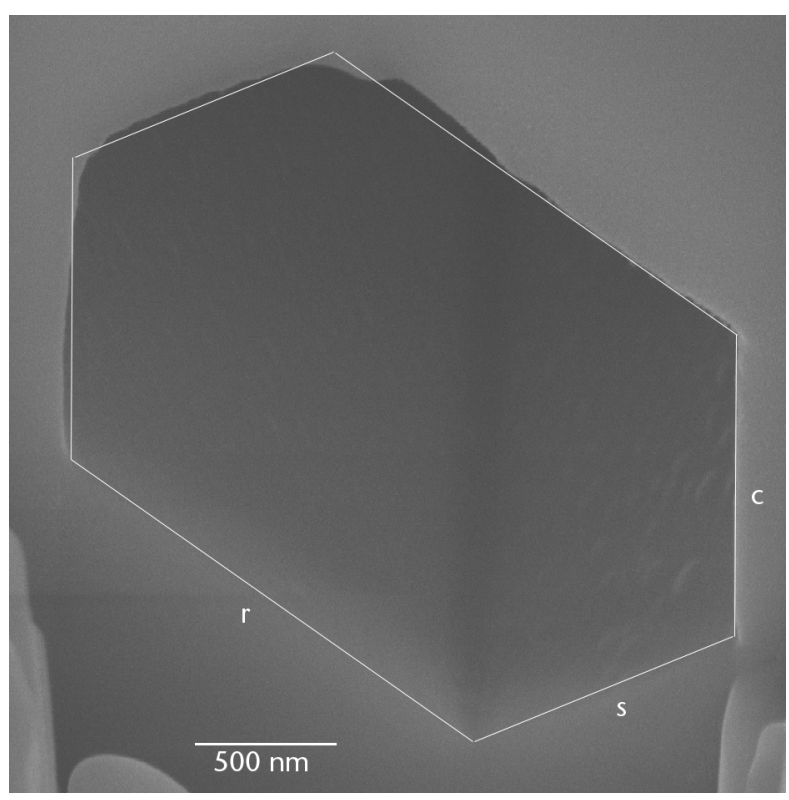

FIgURE 8 Scanning electron micrograph of a cross-section taken perpendicular a $<11 \overline{2} 0>$ direction. Stable channels and features with long axes aligned parallel to a $<11 \overline{2} 0>$ direction develop bounding $\mathbf{c}, \mathbf{r}$, and $\mathbf{s}$ facets, as indicated. 